\title{
Die Bestimmung kleiner Mengen von organischem Stickstoff im Wasser von Binnenseen
}

(Beiträge zur chemischen Wasser- und Abwasseranalyse Nr.5)

\section{Von Marcel Schmid}

Eidg. Anstalt für Wasserversorgung, Abwasserreinigung und Gewässerschutz an der Eidg. Technischen Hochschule, Zürich, Direktion: Prof. Dr. O. Jaag 


\section{Einleitung, Problemstellung}

Beim Studium älterer, aber auch allerneuester limnochemischer Arbeiten stösst man oft auf umfangreiches Zahlenmaterial über die im Wasser enthaltenen chemischen Komponenten, darunter auch jene der Stickstoffgruppe. So haben sich verschiedene Autoren mit der qualitativen und quantitativen Bestimmung der im Wasser gelösten Aminosäuren befasst $[2,8,11,14,25,32]$, und auch Angaben über die anorganischen Stickstoffverbindungen Ammoniak, Nitrit und Nitrat fehlen selten. Einzig über den gesamten gelösten organischen und den an Organismen gebundenen Stickstoff im Seewasser finden sich nur vereinzelt genauere Angaben.

Stickstoff und Phosphor treten im See ungefähr im Verhältnis 10:1 auf. Trotz des im Vergleich zum Phosphor höheren Stickstoffgehalts war es bis heute infolge der vielen Störfaktoren kaum möglich, den organischen Stickstoff mit jener Genauigkeit zu bestimmen, welche für limnologische Zwecke erforderlich ist. Sind in einem städtischen Abwasser Werte von $10 \mathrm{mg} / 1$ organischem Stickstoff nicht selten anzutreffen, so finden wir in einem oligotrophen See Höchstwerte um $0,2 \mathrm{mg} / \mathrm{l}$. Die Störfaktoren, die im Makrobereich kaum bemerkbar sind, führen im Mikrobereich zu ähnlichen analytischen Schwierigkeiten, wie bei der Bestimmung von organischem Phosphor $[21,24]$.

Die Notwendigkeit, eine Gesamtstickstoffmethode zu besitzen, welche die hohen Anforderungen limnologischer Fragestellungen erfüllt, führte uns 1964 dazu, den altbekannten Aufschluss nach KJELDAHL [13] einer systematischen Prüfung zu unterziehen. Es lag uns daran, die Fehlerquellen herauszufinden und womöglich zu eliminieren, ohne dabei eine komplizierte Methode zu entwickeln, sondern eine Lösung zu finden, die es jedem analytischen Wasserlabor erlaubt, ohne allzu grossen Aufwand brauchbare Resultate zu erzielen. 
Im Gegensatz zum Phosphor, der stark in den Energiestoffwechsel der Zellen eingeschaitet ist, hat der Stickstoff vorwiegend Trägerfunktion. Der an das Plankton gebundene Stickstoff, der sogenannte "geformte» oder "partikuläre» Stickstoff, kann daher in gewissen Fällen eine gute Vergleichsbasis mit der berechneten Biomasse des Phytoplanktons liefern [18].

An dieser Stelle möchte ich Herrn Dr. Н.АмвӥнL für die Anregung zur vorliegenden Arbeit sowie für die wertvollen Ratschläge herzlich danken. Mein Dank gilt auch den Herren M. EgGER und B. RIBI, welche die unzähligen Analysen ausgeführt haben.

\section{Literatur}

1883 wurde von J.KJELDAHL eine Analysenmethode zur Stickstoffbestimmung organischer Verbindungen eingeführt [13]. Die Substanz wird mit konzentrierter Schwefelsäure aufgeschlossen, wobei Oxydationsmittel wie $\mathrm{KMnO}_{4}, \mathrm{HClO}_{4}$ oder $\mathrm{H}_{2} \mathrm{O}_{2}$ zugefügt werden, um einen restlosen Abbau zu bewirken und den Stickstoff in Ammoniumsulfat zu überführen. Auch Zugaben von Katalysatoren zum Reaktionsgemisch wie Kupfersulfat, Selen oder Quecksilbersulfat führen unter Umständen zur quantitativen Veraschung.

Die aufgeschlossene Lösung wird dann im Überschuss mit Lauge versetzt und der gebildete Ammoniak mittels Wasserdampfdestillation freigesetzt. Die Bestimmung erfolgt bei hohem Ammoniumgehalt titrimetrisch, bei niederem mit Vorteil kolorimetrisch. Die Kjeldahl-Methode ist nicht allgemein anwendbar [28], sie eignet sich jedoch zur raschen Bestimmung spezifischer Gruppen bei Verbindungen mit niedrigem Stickstoffgehalt, z.B. der Proteine. Als Beispiel schwer aufschliessbarer Substanzen sind vor allem Nitro-, Nitroso- und Azokörper zu nennen; letztere sind im natürlichen Seewasser jedoch kaum anzutreffen.

Die späteren Modifikationen unterscheiden sich von der Originalmethode von KJELDAHL hauptsächlich durch die Katalysatoren für den Aufschluss und die Wahl der Reagenzien für die kolorimetrische Bestimmung. Dem wohlbekannten Reagens nach Nesster, das auch heute noch wegen seiner einfachen Herstellung für die Ammoniumbestimmung an erster Stelle steht $[1,3,7,15,29]$, stehen organische Reagenzien gegenüber. Prochazkova [20] verwendet Bispyrazalon $\left(3,3^{\prime}\right.$-dimethyl-5, $5^{\prime}$ dioxo-1, 1'-diphenyl-(4,4'-bi-2-pyrazolin), das mit Ammonium in Gegenwart von Chloramin T zu einem farbigen Komplex reagiert. Dieser wird mit Trichloräthylen extrahiert. Jönsson [10] arbeitet mit dem von KARLGREN [12] modifizierten Hypochloritphenolat-Reagens, und auch SCHACH [23] empfiehit die IndophenolblauReaktion.

In den letzten Jahren sind verschiedene Arbeiten zur Ultramikrobestimmung von Stickstoff publiziert worden $[29,31]$. Es handelt sich um Entwicklungsarbeiten aus der klinischen Chemie, wo aus geringen Vorlagsmengen kleinste Stickstoffgehalte zu bestimmen sind, ein in der Wasseranalytik selten auftretender Fall. KUISEL [14] hat sich $1935 \mathrm{im}$ Zusammenhang mit der Untersuchung des Zürichsees [8] intensiv mit der Bestimmung von Mikrogramm-Mengen an organischem Stickstoff befasst, musste allerdings zu dieser Zeit unter viel schwierigeren analytischen Bedingungen arbeiten, als dies heute der Fall ist. Er hat jedoch bereits damals grundlegende, auch heute noch 
gültige Vorschriften gegeben. In der neueren Zeit wurde die Stickstoffanalytik speziell von der Meerwasseruntersuchung her frisch beleuchtet. STRICkLAND und PARSONS [30] geben eine Vorschrift über Behandlung der Glasgefässe mit Chromschwefelsäure und betonen, dass die Kjeldahlkolben geschlossen und trocken aufbewahrt werden müssen. Wenn die Analyse nicht nach wenigen Stunden ausgeführt werden kann, so müssen die Proben eingefroren werden. Da ferner die analysenreine Schwefelsäure unterschiedliche Mengen an Stickstoff enthält, empfehlen die Autoren, von mehreren Säuren den Stickstoffgehalt (= Blindwert) zu bestimmen und jeweils nur jene mit dem tiefsten Gehalt zum Aufschluss der Proben zu verwenden. Für die Bestimmung werden nur $25 \mathrm{ml}$ Wasser verwendet, und die Aufschlusszeit beträgt 60 Minuten bei $200^{\circ} \mathrm{C}$.

Rотн [22] hat speziell die auftretenden Fehler beim Kjeldahlaufschluss kritisch untersucht und dabei sehr wertvolle Feststellungen gemacht. Um den Stickstoff genau erfassen zu können, muss man den Blindwert auf ein konstantes Minimum herabsetzen. Den grössten Anteil an Stickstoff erhält der Blindwert aus der verwendeten Schwefelsäure, und zwar abhängig von der Herkunft, der Art der Aufbewahrung und dem Eintauchen von Pipetten. Roтн gibt eine Vorschrift zur Reinigung der Schwefelsäure an. Ein weiterer Blindwert, der nach Ansicht des Autors erheblichen Schwankungen unterliegt, bildet sich im Verlauf des Aufschlusses. Die Schwefelsäuredämpfe können basische Verbindungen (Ammoniak) aus der Luft binden. Bei der Kondensation gelangen so unkontrollierbare Mengen an Stickstoff zum Aufschlussgut. Als Gegenmassnahme wird ein Aufsatz auf den Kjeldahlkolben gesetzt, der genügend lang ist, um als Kühler zu wirken. Am Ende des Rohrs dient ein U-Bogen, der mit verdünnter Schwefelsäure (als Sperrflüssigkeit) gefüllt ist, als Abschluss gegen die Aussenluft. Mit Hilfe dieser Vorsichtsmassnahme gelangt Rотн zu befriedigenden Resultaten, speziell beim Aufschluss von organischem und biologischem Material. ProchazKova [19] untersuchte den Einfluss der Nitrite und Nitrate auf die Bestimmung des organischen Stickstoffs im Wasser. Nitrite üben erst einen störenden Einfluss aus, wenn ihre Konzentration 10mal höher liegt als die des organischen Stickstoffs, wogegen die Nitrate zu niedrige Stickstoffwerte zur Folge haben. Der störende Einfluss des Nitrats hängt auch von der Schwefelsäuremenge, von Chloridionen und von reduzierenden Stoffen ab [4]. Die Nitrate bilden mit der Schwefelsäure ein Oxydationsmilieu, wodurch das entstehende Ammonium zu höherwertigen Stickstoffverbindungen oxydiert wird. Durch Zugabe von Bisulfit soll diese Oxydationswirkung verringert werden. Dudova [5] befasst sich ebenfalls mit der Bestimmung von organischem Stickstoff in Gegenwart von mineralischem Stickstoff. Der Nitrat-N-Anteil wird hier in saurem Milieu mit $\mathrm{FeSO}_{4}$ zu NO reduziert, welches dann durch Kochen entfernt wird. Auch Starikova und Mitarbeiter [27] beheben den $\mathrm{NO}_{2}-\mathrm{N}$ - und den $\mathrm{NO}_{3}-\mathrm{N}$ Einfluss durch Zugabe von Eisensulfat. Diese Methoden führen jedoch nur dann zum Ziel, wenn kein $\mathrm{H}_{2} \mathrm{O}_{2}$ als Oxydationsmittel verwendet wird. McKENZIE und Mitarbeiter [16] studierten den Aufschluss und untersuchten dabei die Katalysatoreinwirkung, den Einfluss der oxydierenden Agentien und die Temperaturbedingungen. Als geeignetster Katalysator wird Quecksilber empfohlen, wobei zur Verhinderung der Bildung von $\mathrm{HgO}$, welches Ammonium absorbieren kann, der Natronlauge $\mathrm{Na}_{2} \mathrm{~S}$ oder $\mathrm{Na}_{2} \mathrm{~S}_{2} \mathrm{O}_{3}$ beigegeben werden müssen. Von den oxydierenden Agentien wird nur $\mathrm{H}_{2} \mathrm{O}_{2}$ als brauchbar befunden. Die anderen Mittel können Ammonium bis $z \mathrm{~N}_{2}$ oxydieren. 
Temperaturen zwischen $380-390^{\circ} \mathrm{C}$ geben normalerweise bei den meisten natürlichen Stickstoffverbindungen einen quantitativen Aufschluss. FLECK und Munro [6] geben in ihrer guten Übersicht über die Bestimmung von organischem Stickstoff in biologischem Material als günstigste Aufschlussbedingungen eine Temperatur um $370-410^{\circ} \mathrm{C}$ und Quecksilber als Katalysator an.

\section{Erfahrungen und praktische Prüfung der Methode}

\subsection{Herstellung von $N$-freiem $W$ asser}

Zur Reinigung der zur N-Bestimmung verwendeten Glasgefässe und zur Herstellung der Typreihe und der Reagenzien darf nur N-freies Wasser verwendet werden. Es ist deshalb notwendig, davon einen genügenden Vorrat $z u$ halten.

Destilliertes Wasser wird unter Zusatz von $3 \mathrm{ml} / \mathrm{l}$ alkalischer $\mathrm{KMnO}_{4}$-Lösung $\left(10 \mathrm{~g} \mathrm{KMnO}_{4}+50 \mathrm{~g} \mathrm{NaOH}\right.$ in $1000 \mathrm{ml}$ destilliertem Wasser) ein zweites Mal destilliert, wobei der erste $\mathrm{N}$-haltige Anteil verworfen wird.

Da es kaum möglich ist, ein Laboratorium völlig staub- und ammoniakfrei zu halten, muss die Apparatur vor Einflüssen dieser Art geschützt werden. Hierzu hat sich die in Abb. 1 dargestellte Destillationsanordnung bewährt.

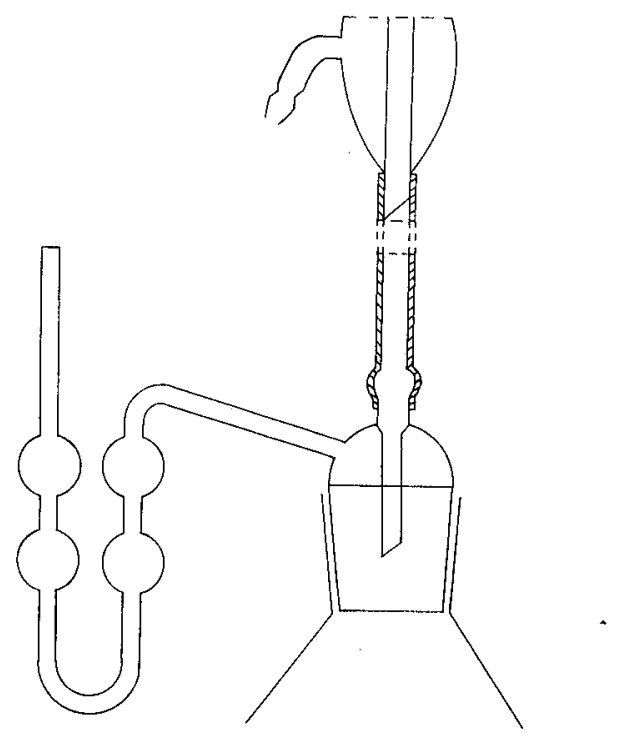

Abb.1. Verschluss der Auffangflasche während der Herstellung von stickstofffreiem Wasser.

Das Destillat gelangt ohne Luftberührung in die Auffangflasche. Zur Sperrung dient ein U-Rohr, das mit verdünnter Schwefelsäure gefüllt ist. Wird diese Vorsichtsmassnahme eingehalten, so erhält man einen Nachlauf, der gemäss Abb.2 praktisch $\mathrm{N}$-frei ist (unter der Nachweisgrenze von $10 \mu \mathrm{g} / 1 \mathrm{NH}_{4}-\mathrm{N}$ ). 


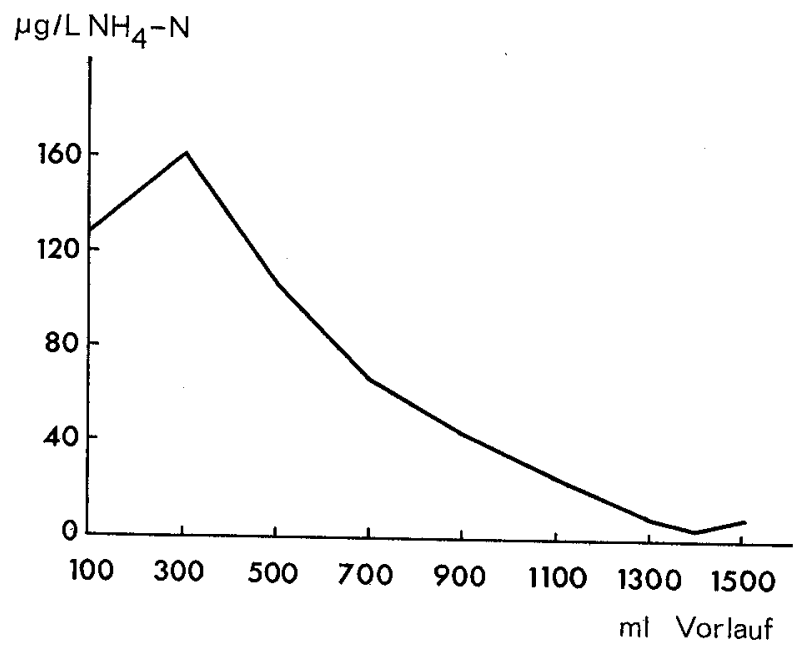

Abb.2. Abnahme des Stickstoffgehalts in Funktion der Vorlaufmenge.

\subsection{Reinigung der Schwefelsäure, Vergleich verschiedener Fabrikate, Aufbewahrung der Säure}

Roth [22] gibt eine Vorschrift zur Reinigung der für den Aufschluss verwendeten Schwefelsäure. Die Säure wird mit Zinkpulver erhitzt. Vor der nachfolgenden Vakuumdestillation wird etwas Ammoniumsulfat zugegeben; der Vorlauf wird verworfen. Verglichen mit der unbehandelten Schwefelsäure lieferte die von uns auf solche Art gereinigte Säure keine wesentlich tieferen Blindwerte (Tab.1).

Schwefelsäure 3 ergab beim erstmaligen Öffnen der Flasche einen Durchschnittsblindwert (erhalten aus 16 Proben) von $1,95 \mathrm{mg} / 1 \mathrm{NH}_{4}-\mathrm{N}$. Nach 15 Tagen und mehrmaligem Öffnen der Flasche war der Wert auf 3,1 mg/1 $\mathrm{NH}_{4}-\mathrm{N}$ angestiegen, und am 20. Tag war er bereits bei 4,2 mg/l angelangt. Diese Zahlen zeigen, dass durch Eintauchen von Pipetten $\mathrm{N}$-haltige Verunreinigungen in die Säure eingeschleppt werden.

Tab.1. Stickstoffgehalt verschiedener Schwefelsäuren.

\begin{tabular}{|c|c|c|}
\hline & $\begin{array}{l}\text { Reinheitsangabe } \\
\text { der Herstellerfirma }\end{array}$ & $\begin{array}{l}\text { Bestimmter } \\
\text { Stickstoffwert }\end{array}$ \\
\hline $\begin{array}{l}\text { Schwefelsäure 1 } \\
\text { Schwefelsäure 2 }\end{array}$ & $\max .5 \mathrm{mg} / 1$ & $2,2 \mathrm{mg} / \mathrm{l}$ \\
\hline gereinigt nach Rотн & $\max .5 \mathrm{mg} / 1$ & $3,0 \mathrm{mg} / \mathrm{l}$ \\
\hline Schwefelsäure 3 . . . . & $1,84 \mathrm{mg} / \mathrm{l}$ & $1,95 \mathrm{mg} / \mathrm{I}$ \\
\hline
\end{tabular}

Jede neue, für die Stickstoffbestimmung verwendete Schwefelsäure wird von uns auf ihren $\mathrm{N}$-Gehalt geprüft. Liegt der Wert zwischen 2 und $3 \mathrm{mg} / \mathrm{l}$, so wird der Inhalt auf 100-ml-Flaschen aus Jenaer Glas G20 verteilt. Die Schwefelsäure ist so nach 2-3maligem Öffnen der Flasche aufgebraucht. 
3.3. Fremdeinflüsse beim Abdampfen und Aufschliessen von Wasserproben. N-Zunahme und $N$-Verluste

In den folgenden Ausführungen werden der besseren Übersicht wegen sämtliche Angaben in Mikrogramm pro Liter gemacht.

Eine Prüfung des Abdampfvorganges ist recht schwierig. Damit sicher keine NVerluste auftreten, muss die Schwefelsäure schon vor dem Sieden zur Probe zugesetzt werden. Werden nun 100, 200 oder $300 \mathrm{ml}$ Seewasser abgedampft und die Kolben beim ersten Auftreten von $\mathrm{SO}_{3}$-Dämpfen von den Brennern entfernt, so wird der Blindwert durch die jeweils herrschende Endtemperatur beeinflusst, da unterschiedliche Mengen an organischem Stickstoff, von der Schwefelsäure herrührend, in Ammoniumsulfat umgewandelt werden. Ein Vergleich zwischen einzelnen Proben ist daher kaum möglich. Es ist jedoch mit ziemlicher Sicherheit anzunehmen, dass bei den beim Abdampfen vorherrschenden sauren Bedingungen nicht mit Ammoniumverlusten zu rechnen ist.

Behandlung von Ammoniumtestlösungen : $200 \mathrm{ml} \mathrm{N}$-freies Wasser mit $100 \mu \mathrm{g} / \mathrm{l}$ $\mathrm{NH}_{4}-\mathrm{N}+2 \mathrm{ml}$ konz. $\mathrm{H}_{2} \mathrm{SO}_{4}$ wurden abgedampft und anschliessend 30 Minuten bei einer Temperatur von ungefähr $320^{\circ} \mathrm{C}$ aufgeschlossen. Die Resultate sind in Tab.2 wiedergegeben.

Tab. 2. Aufschluss von $\mathrm{NH}_{\mathbf{4}}-\mathrm{N}$-Standardlösungen.

\begin{tabular}{llllll}
\hline $\begin{array}{l}\text { Sollwert } \\
{[\mu \mathrm{g} / 1]}\end{array}$ & $\begin{array}{l}\text { Gefundener } \\
\text { Wert } \\
{[\mu \mathrm{g} / \mathrm{l}]}\end{array}$ & $\begin{array}{l}\text { Differenz } \\
{[\mu \mathrm{g} / 1]}\end{array}$ & $\begin{array}{l}\text { Sollwert } \\
{[\mu \mathrm{g} / 1]}\end{array}$ & $\begin{array}{l}\text { Gefundener } \\
\text { Wert } \\
{[\mu \mathrm{g} / 1]}\end{array}$ & $\begin{array}{c}\text { Differenz } \\
{[\mu \mathrm{g} / \mathrm{l}]}\end{array}$ \\
\hline 100 & 100 & 0 & 100 & 94 & -6 \\
100 & 88 & -12 & 100 & 90 & -10 \\
100 & 78 & -22 & 100 & 86 & -14 \\
100 & 80 & -20 & 100 & 88 & -12 \\
100 & 92 & -8 & 100 & 94 & -6 \\
\hline
\end{tabular}

Dieser Versuch zeigt, dass beim Aufschluss recht grosse Verluste auftreten können. Anderseits fanden wir im Zusammenhang mit der Herstellung von $\mathrm{N}$-freiem Wasser (s. S. 247) und bei Aufschlussversuchen die Theorie von Rотн bestätigt, nach der die Schwefelsäuredämpfe, aber auch das destillierte Wasser stickstoffhaltige basische Verbindungen aus der Luft aufnehmen können. Gelangen die Dämpfe wieder zum Aufschlussgut, so erhält man zu hohe Werte, wie Tab. 3 zeigt.

10 Proben von je $2 \mathrm{ml}$ konz. Schwefelsäure wurden 30 Minuten im Kjeldahlkolben bei Aufschlusstemperatur erhitzt. Während die Normalwerte bei ungefähr $5 \mu \mathrm{g} / 2 \mathrm{ml}$ Schwefelsäure liegen, lieferte diese Probenreihe erheblich höhere Werte.

Tab. 3. 10 Blindwerte einer konzentrierten Schwefelsäure.

\begin{tabular}{lllllllllll}
\hline Gefundener Wert $[\mu \mathrm{g} / 2 \mathrm{ml}]:$ & 26 & 13 & 14 & 10 & 16 & 9 & 19 & $\mathbf{1 4}$ & 16 & 11 \\
\hline
\end{tabular}


Wie nachträglich festzustellen war, enthielt die Atmosphäre infolge verschiedener Laboratoriumsversuche während des Aufschlusses und der Destillation etwas Ammonium, was diese hohen Blindwerte erklärt.

Da nach unseren Erfahrungen einerseits $\mathrm{NH}_{4}-\mathrm{N}$-Verbindungen während des Aufschlusses entweichen können, anderseits durch die sauren $\mathrm{SO}_{3}-$ Dämpfe $\mathrm{NH}_{4}-\mathrm{N}$ aus der Luft gebunden werden kann, suchten wir nach einem Ausweg, um beide Störungen auszuschalten. Dies geschieht vorteilhaft durch Sperrung nach dem Vorschlag von Rотн [22], jedoch in doppelter Ausführung (Abb.3). Als Sperrflüssigkeit verwenden wir 1prozentige Schwefelsäure, wobei die äussere Flüssigkeit Fremdeinflüsse von aussen abhalten soll, die innere Sperrung evtl. auftretende Verluste zurückhalten muss. Diese letztere Flüssigkeit wird nach beendetem Aufschluss in den Kjeldahlkolben gegeben. Nach unseren Erfahrungen an rund 3000 Seewasserproben wären diese Vorsichtsmassnahmen bei weitem nicht immer notwendig. Da man jedoch vor Inangriffnahme einer Stickstoffbestimmung normalerweise nicht wissen kann, ob die Atmosphäre des Labors genügend rein ist, empfehlen wir dieses Vorgehen für jeden Aufschluss. Um zu vergleichen, ob die Sperrung mit U-Rohren ausreicht, verwendeten wir versuchsweise U-Rohre, welche zur besseren Absorption mit Glasperlen gefüllt waren. Hier wie auch bei Versuchen, bei denen Rückflusskühler auf die Kjeldahlkolben gesetzt wurden, erhielten wir viel zu hohe Werte. Vermutlich wirkte sich die grössere Oberfläche ungünstig aus, indem die heissen $\mathrm{SO}_{3}$-Dämpfe mit mehr Luft in Berührung kamen.

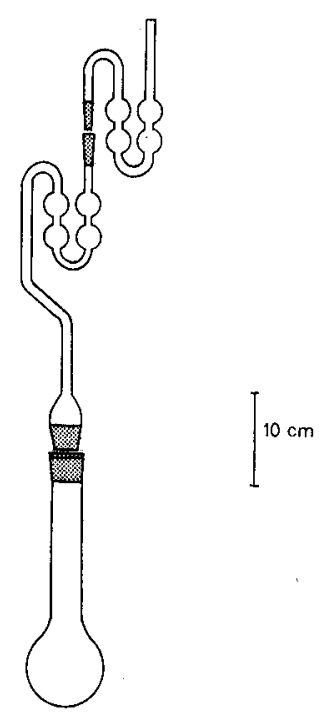

Abb. 3. Verschluss der Kjeldahlkolben während des Aufschlussvorgangs mit zweifachem U-Rohr.

Nachdem die Doppelaufsätze 3 Tage lang gebraucht wurden, ohne die Sperrflüssigkeit in den äusseren U-Rohren zu wechseln, bestimmten wir darin den $\mathrm{NH}_{4}-\mathrm{N}-$ Gehalt (Tab.4). 
Tab.4. Stickstoffgehalt von je $5 \mathrm{ml}$ 1 prozentiger Schwefelsäure, die 3 Tage zur Sperrung gebraucht und anschliessend auf $100 \mathrm{ml}$ verdünnt wurden.

\begin{tabular}{lllllllllllllll}
\hline Zunahme an $\mathrm{NH}_{4}-\mathrm{N}[\mu \mathrm{g} / 1]:$ & 0 & 30 & 10 & 25 & 0 & 5 & 40 & 0 & 0 & 0 & 5 & 0 \\
\hline
\end{tabular}

Auch diese Zahlen veranschaulichen, dass eine Störung von aussen her nicht immer eintreten muss, dass sie jedoch in einzelnen Fällen zu bedeutenden Fehlern führen kann.

Einen weiteren, zwar banalen, aber wesentlichen Störfaktor, an den sicher nicht ohne weiteres gedacht wird, bildet das Ammonium an den Hänđen des Analytikers. Bei den verschiedenen Operationen besteht grosse Gefahr, dass nasse Glasteile mit der Hand in Berührung kommen und die so verunreinigte Flüssigkeit nachher zum Aufschlussgut gelangt. Nach Oro und Skewes [17] und Hamilton [9] sind neben Harnstoff und Ammoniak vor allem Aminosäuren wie Serin, Glycin und Lysin, welche $\mathrm{NH}_{4}-\mathrm{N}$-Anteile liefern, zu nennen. Es gilt daher, während der ganzen Analyse sämtliche kritischen Berührungsstellen an den Glasgefässen besonders sorgfältig zu beachten. Wie auch Tab. 5 zeigt, ist es durchaus möglich, dass durch Schweiss der Hand Ammonium in die Proben geschleppt wird.

Tab. 5. Eine ungewaschene, eine gewaschene und eine mit Handschuhen geschützte Hand wurden mit je $100 \mathrm{ml} \mathrm{N}$-freiem Wasser gespült. Anschliessend Zugabe von $4 \mathrm{ml}$ Nessler-Reagens und Messung im Photometer.

Extinktion $[100 \mathrm{~mm}, 440 \mathrm{~nm}$ ]

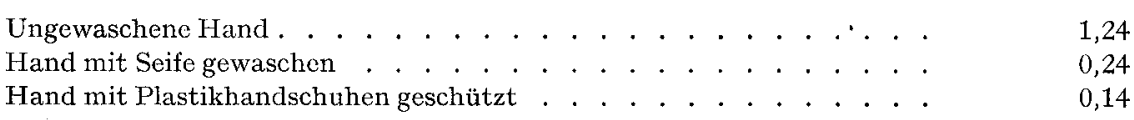

Die Störung durch Ammonium an den Händen kann somit durch häufiges Händewaschen mit Seife wesentlich verringert werden. Das beste Resultat erzielt man mit Handschuhen (Wegwerfhandschuhen aus dünner Plastikfolie), wobei das Arbeiten allerdings etwas erschwert wird. Allgemein gilt, dass überall dort, wo mit unverschlossenen Kjeldahlkolben gearbeitet wird, jegliche Ausdünstung, faulige Gerüche usw. zu Fehlern führen.

\subsection{Fehler bei der Destillation}

Zur Abklärung, ob zur Absorption des freiwerdenden Ammoniums destilliertes Wasser oder verdünnte Schwefelsäure nötig sind, destillierten wir Proben mit $300 \mu \mathrm{g} / 1$ $\mathrm{NH}_{4}-\mathrm{N}$ in verschiedene Vorlagen.

Aus Tab. 6 ist ersichtlich, dass destilliertes Wasser das freiwerdende Ammonium ungenügend absorbiert. Verwendet man hingegen eine schwachsaure Lösung und destilliert etwa $70 \mathrm{ml}$ in die Vorlage, welche wiederum gegen die Aussenluft abgeschlossen ist (Abb.4), so werden $100 \%$ des vorgelegten Ammoniums gefunden.

Vor der Destillation der Proben wird die Apparatur mit N-freiem Wasser gefüllt, pro Liter Wasser $2 \mathrm{ml}$ kaltgesättigte Sodalösung zugefügt und anschliessend so lange 
Tab.6. Destillation von je $300 \mu \mathrm{g} / 1 \mathrm{NH}_{4}-\mathrm{N}$ in verschieden grosse neutrale und saure Vorlagen.

\begin{tabular}{|c|c|c|}
\hline Vorlage & \multirow{2}{*}{$\frac{\mathrm{NH}_{4}-\mathrm{N}[\mu \mathrm{g} / \mathrm{l}]}{300}$} & \multirow{2}{*}{$\frac{\text { Gefundener Wert }[\mu \mathrm{g} / \mathrm{l}}{280}$} \\
\hline $10 \mathrm{ml}$ dest. Wasser & & \\
\hline $30 \mathrm{ml}$ dest. Wasser & 300 & 285 \\
\hline $\begin{array}{l}50 \mathrm{ml} \text { dest. Wasser } \\
10 \mathrm{ml} \text { dest. Wasser }\end{array}$ & 300 & 285 \\
\hline $\begin{array}{l}+0,1 \mathrm{ml} 10 \text { prozentige } \mathrm{H}_{2} \mathrm{SO}_{4} \\
30 \mathrm{ml} \text { dest. Wasser }\end{array}$ & 300 & 302 \\
\hline $\begin{array}{l}+0,1 \mathrm{ml} 10 \text { prozentige } \mathrm{H}_{2} \mathrm{SO}_{4} \\
50 \mathrm{ml} \text { dest. Wasser }\end{array}$ & 300 & 301 \\
\hline$+0,1 \mathrm{ml} 10$ prozentige $\mathrm{H}_{2} \mathrm{SO}_{4} \ldots \ldots$ & 300 & 285 \\
\hline
\end{tabular}

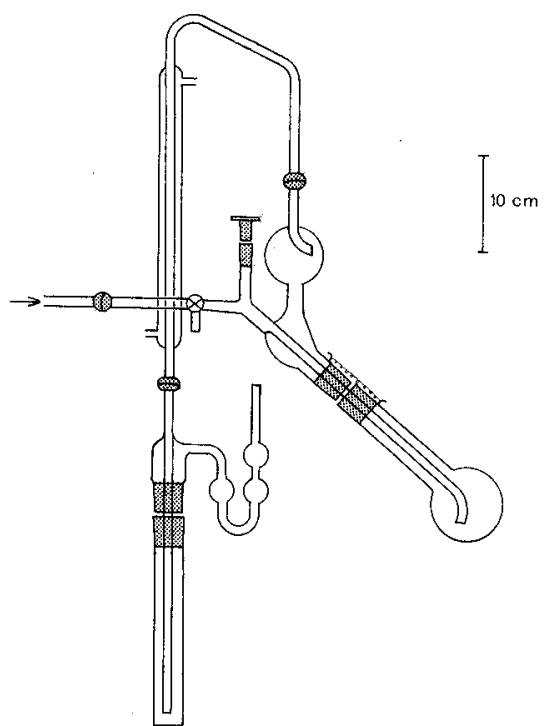

Abb.4. Apparatur zur Destillation der aufgeschlossenen Proben. Auffanggefäss mit Sperrvorrichtung (U-Rohr).

vordestilliert, bis der Dampf $\mathrm{N}$-frei anfällt. Um ganz sicher zu sein, dass die ganze Apparatur als $\mathrm{N}$-frei angesehen werden darf, empfiehlt es sich, vor der Destillation der Proben den Kjeldahlkolben an der Apparatur auszuwechseln, Lauge zuzugeben (gemäss Vorschrift auf S. 263) und etwa $70 \mathrm{ml}$ zu destillieren. Dann kann mit der Destillation der Proben begonnen werden.

\subsection{Katalysatoren; störender Einfluss von Nitrat}

Im Zusammenhang mit dem evtl. störenden Einfluss der Nitrate wurde die Katalysatorenfrage geprüft.

Je $100 \mu \mathrm{g} / 1 \mathrm{NH}_{4}-\mathrm{N}, 2 \mathrm{ml}$ konz. Schwefelsäure, Quecksilbersulfat als Katalysator oder $\mathrm{H}_{2} \mathrm{O}_{2}$ als Oxydationsmittel wurden mit steigenden Nitratmengen versetzt und 30 Minuten erwärmt. Die Ergebnisse sind aus Tab.7 ersichtlich. 
Tab. 7. Der Einfluss der Nitrate im Zusammenhang mit Katalysator oder Oxydationsmittel

\begin{tabular}{llcr}
\hline Katalysator bzw. Oxydationsmittel & $\begin{array}{l}\mathrm{NH}_{4}-\mathrm{N} \text { vorgelegt } \\
{[\mu \mathrm{g} / 1]}\end{array}$ & $\begin{array}{l}\mathrm{NO}_{3}-\mathrm{N} \\
{[\mu \mathrm{g} / \mathrm{I}]}\end{array}$ & $\begin{array}{r}\mathrm{NH}_{4}-\mathrm{N} \text { gefunden } \\
{[\mu \mathrm{g} / 1 \hat{=} \%]}\end{array}$ \\
\hline $\mathrm{Hg}$-Sulfat, 1.prozentig & 100 & 0 & 102 \\
$2 \mathrm{ml}$ konz. $\mathrm{H}_{2} \mathrm{SO}_{4}$ & 100 & 200 & 86 \\
& 100 & 400 & 54 \\
& 100 & 600 & 36 \\
& 100 & 800 & 18 \\
$\mathrm{H}_{2} \mathrm{O}_{2}, 30$ prozentig & 100 & 0 & 101 \\
$2 \mathrm{ml}$ konz. $\mathrm{H}_{2} \mathrm{SO}_{4}$ & 100 & 200 & 97 \\
& 100 & 400 & 97 \\
& 100 & 600 & 73 \\
$2 \mathrm{ml}$ konz. $\mathrm{H}_{2} \mathrm{SO}_{4}$ & 100 & 800 & 68 \\
& 100 & 0 & 98 \\
& 100 & 200 & $\mathbf{1 0 2}$ \\
& 100 & 400 & 85 \\
& 100 & 600 & 80 \\
& 100 & 800 & 88 \\
\hline
\end{tabular}

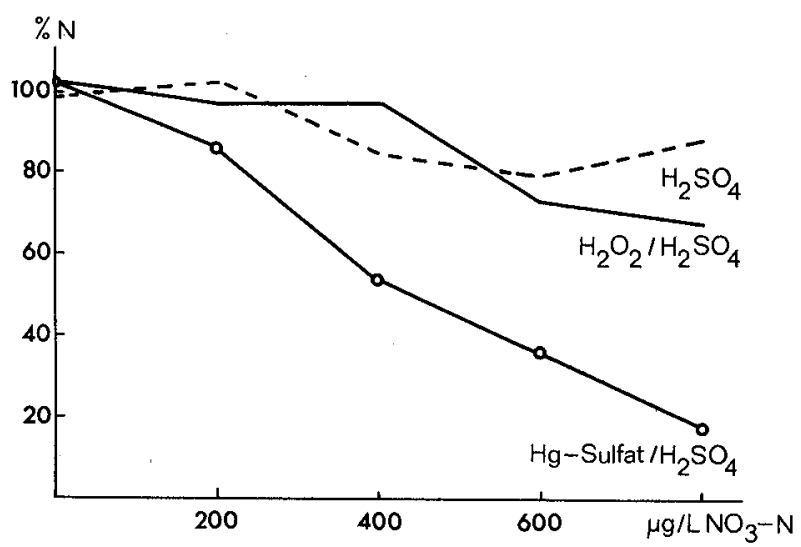

Abb. 5. Störung durch Nitrat bei Anwendung von Katalysator oder Oxydationsmittel

Die Werte aus Tab. 7 sind in Abb. 5 graphisch dargestellt. Solange der Nitratstickstoff nicht entfernt wird, kommt Quecksilbersulfat als Katalysator nicht in Frage. Verwendet man $\mathrm{H}_{2} \mathrm{O}_{2}$ als Oxydationsmittel, so scheint ein grösserer Fehler erst bei Gehalten zwischen 400 und $500 \mu \mathrm{g} / 1$ Nitratstickstoff einzutreten, ein Wert, der bei oligotrophen Seen eher an der oberen Grenze liegt. Eine unterschiedliche Abnahme tritt auch ein, wenn nur Schwefelsäure allein verwendet wird. Dass höhere Nitratwerte eindeutig stören und Verluste bis zu 100\% verursachen können, zeigt folgendes Beispiel: Oscillatoria rubescens, in Kulturmedien gezüchtet, wurde regelmässig geerntet, aufgeschlossen und darin der geformte Stickstoffgehalt bestimmt. Die Nährlösung enthielt ursprünglich $84 \mathrm{mg} / 1 \mathrm{NO}_{3}-\mathrm{N}$, und der Algenanteil (Trockensubstanz), der etwa um $5 \mathrm{mg} / \mathrm{l} \mathrm{lag}$, was $0,5 \mathrm{mg} / 1$ Stickstoff entsprechen würde, nahm beständig $\mathrm{zu}$. 
Trotzdem konnten wir überhaupt keinen organischen Stickstoff nachweisen, da anscheinend beim Aufschluss die gesamte Menge zu flüchtigen Verbindungen oxydiert wurde. In diesem Fall erhielten wir durch Filtration der Nährlösung durch ein Membranfilter mit einer Porenweite von $0,45 \mu \mathrm{m}$ und anschliessendem separatem Aufschliessen des abgewaschenen Filterrückstands gute Resultate. Da es sich um leicht zerstörbares Material handelte und ein Katalysator oder $\mathrm{H}_{2} \mathrm{O}_{2}$ kaum nötig waren, gelangten wir auch mit Hilfe der Vorschrift von PRochazkova [19] mit Schwefelsäure und Natriumbisulfit als schwachem Reduktionsmittel zu befriedigenden Ergebnissen. Dass der $\mathrm{NO}_{3}-\mathrm{N}$-Einfluss von der Aufschlussdauer abhängt, veranschaulicht Tab. 8 .

Tab.8. Der Einfluss der Nitrate in Funktion der Aufschlussdauer.

\begin{tabular}{llll}
\hline $\begin{array}{l}\mathrm{NH}_{4}-\mathrm{N} \\
{[\mu \mathrm{g} / \mathrm{l}]}\end{array}$ & $\begin{array}{l}\mathrm{NO}_{3}-\mathrm{N} \\
{[\mu \mathrm{g} / 1]}\end{array}$ & $\begin{array}{l}\text { Aufschlusszeit } \\
{[\text { Minuten }]}\end{array}$ & $\begin{array}{l}\text { Gefundener Wert + Blindwert } \\
{[\mu \mathrm{g} / 1]}\end{array}$ \\
\hline 100 & - & 5 & 150 \\
100 & - & 10 & 160 \\
100 & - & 15 & 150 \\
100 & - & 20 & 140 \\
100 & - & 25 & 150 \\
100 & - & 30 & 150 \\
100 & 500 & 5 & 140 \\
100 & 500 & 10 & 140 \\
100 & 500 & 20 & 140 \\
100 & 500 & 25 & 140 \\
100 & 500 & 30 & 130 \\
100 & 500 & 120 \\
\hline
\end{tabular}

Wird mit $2 \mathrm{ml}$ konz. $\mathrm{H}_{2} \mathrm{SO}_{4}$ und 3 Tropfen $\mathrm{H}_{2} \mathrm{O}_{2}$ aufgeschlossen, so liegen die Werte innerhalb der Fehlergrenze. Wird derselbe Versuch mit Zusatz von $500 \mu \mathrm{g} / \mathrm{l}$ $\mathrm{NO}_{3}-\mathrm{N}$ durchgeführt, so tritt eine Abnahme des Ammoniumgehalts erst nach 20 Minuten auf. Aufschlussversuche mit $100 \mu \mathrm{g} / \mathrm{l}$ Glycin und Tryptophan ergaben, dass nach 10-15 Minuten für beide Verbindungen der gesamte organische Stickstoffanteil abgebaut ist.

Zur Abklärung, wie gross der Nitratfehler sich bei Seewasserproben auswirkt, wurde noch der folgende Versuch durchgeführt: $\mathrm{Zu}$ je $100 \mathrm{ml}$ filtriertem Vierwaldstätterseewasser aus $20 \mathrm{~m}$ Tiefe mit einem Nitratstickstoffgehalt von $330 \mu \mathrm{g} / 1$ wurden zusätzlich 500 und $1000 \mu \mathrm{g} / \mathrm{l} \mathrm{NO}_{3}-\mathrm{N}$ zugegeben. Der Ausgangsnitratgehalt lag somit nach den gemachten Erfahrungen in einem Bereich, in welchem keine grossen Verluste eintreten. Die Proben wurden mit $2 \mathrm{ml}$ konz. Schwefelsäure und 3 Tropfen $\mathrm{H}_{2} \mathrm{O}_{2} 30$ Minuten bei $310^{\circ} \mathrm{C}$ aufgeschlossen. Anschliessend wurde darin der $\mathrm{NH}_{4}-\mathrm{N}$-Gehalt bestimmt (Tab.9).

Hieraus ist ersichtlich, dass im Gegensatz zu den künstlich hergestellten Proben aus destilliertem Wasser und $\mathrm{NH}_{4}-\mathrm{N}$, wo sich der störende Nitratgehalt bereits von $500 \mu \mathrm{g} / \mathrm{l}$ an bemerkbar macht (Tab. 7 und Abb.5), bei Seewasserproben erst höhere Nitratgehalte zu Verlusten führen. Liegen Nitratgehalte von etwas über $1000 \mu \mathrm{g} / 1 \mathrm{vor}$, so kann durch Zusatz von $1 \mathrm{ml} 1$ prozentigem Natriumbisulfit (beim Eindampfen) der Verlust nahezu eliminiert werden (Tab.10). 
Tab. 9. Der Einfluss von Nitrat auf Seewasserproben.

$\left.\begin{array}{lccc}\hline \text { Filtriertes Seewasser }[\mathrm{ml}] & \mathrm{NO}_{3}-\mathrm{N}[\mu \mathrm{g} / \mathrm{l}] & \mathrm{NH}_{\mathbf{4}}-\mathrm{N}_{\text {gefunden }[\mu \mathrm{g} / \mathrm{l}]} \\ \hline 100 & 330 & 120 & \\ 100 & 330 & 110 & \varnothing 110 \\ 100 & 330 & 100\end{array}\right\}$

Tab.10. Verminderung des Nitrateinflusses auf Seewasserproben mittels Natriumbisulfit und ohne $\mathrm{H}_{2} \mathrm{O}_{2}$ als Oxydationsmittel.

\begin{tabular}{|c|c|c|c|c|}
\hline $\begin{array}{l}\text { Filtriertes Seewasser } \\
{[\mathrm{ml}]}\end{array}$ & $\begin{array}{l}\mathrm{NO}_{3}-\mathrm{N} \\
{[\mu \mathrm{g} / 1]}\end{array}$ & $\begin{array}{l}\mathrm{NaHSO}_{3}, 1 \text { prozentig } \\
{[\mathrm{ml}]}\end{array}$ & $\begin{array}{l}\mathrm{NH}_{4}-\mathrm{N} \\
{[\mu \mathrm{g} / 1]}\end{array}$ & gefunden \\
\hline 100 & 330 & 1 & 120 & \multirow{3}{*}{$\varnothing 120$} \\
\hline 100 & 330 & 1 & 120 & \\
\hline 100 & 330 & 1 & 120 & \\
\hline 100 & 830 & 1 & 120 & \multirow{3}{*}{$\varnothing 120$} \\
\hline 100 & 830 & 1 & 110 & \\
\hline 100 & 830 & 1 & 120 & \\
\hline 100 & 1330 & 1 & 120 & \multirow{5}{*}{$\varnothing 110$} \\
\hline 100 & 1330 & 1 & 120 & \\
\hline 100 & 1330 & 1 & 120 & \\
\hline 100 & 1330 & 1 & 90 & \\
\hline 100 & 1330 & 1 & 100 & \\
\hline
\end{tabular}

Wird Quecksilber als Katalysator verwendet, so kann durch Zugabe von $\mathrm{NaCl}$ der Fehler im Bereich bis zu $1000 \mu \mathrm{g} / 1$ wesentlich verringert werden (Tab.11).

Tab.11. Verminderung des störenden Einflusses von Nitrat mittels Natriumchlorid, wenn Quecksilber als Katalysator verwendet wird.

\begin{tabular}{lllll}
\hline $\begin{array}{l}\mathrm{NH}_{4}-\mathrm{N} \\
{[\mu \mathrm{g} / \mathrm{l}]}\end{array}$ & $\begin{array}{l}\mathrm{NO}_{3}-\mathrm{N} \\
{[\mu \mathrm{g} / 1]}\end{array}$ & $\begin{array}{l}\mathrm{NaCl}, 10 \text { prozentig } \\
{[\mathrm{ml}]}\end{array}$ & $\begin{array}{l}\mathrm{NH}_{4}-\mathrm{N}, \text { gefunden } \\
{[\mu \mathrm{g} / 1]}\end{array}$ & $\begin{array}{l}\mathrm{NH}_{4}-\mathrm{N}, \text { gefunden } \\
\text { ohne NaCl-Zusatz }\end{array}$ \\
\hline 100 & 0 & 1 & 100 & 102 \\
100 & 200 & 1 & 84 & 86 \\
100 & 400 & 1 & 86 & 54 \\
100 & 600 & 1 & 80 & 36 \\
100 & 800 & 1 & 92 & 18 \\
\hline
\end{tabular}

Da jedoch unter den von uns gewählten Aufschlussbedingungen (20 Minuten bei $310^{\circ} \mathrm{C}$ und Quecksilber als Katalysator) für Tryptophan nur 50-60\% des theoretischen Stickstoffgehalts gefunden wurde (für Glycin 100\%) und auch die Streuung höher lag, kam für uns dieser Katalysator nicht in Frage. 


\subsection{Störung durch Nitrit}

Nach Prochazkova [19] stören auch grössere Nitritkonzentrationen nicht, da sie sich während des sauren Abdampfvorgangs zersetzen und verflüchtigen.

Auch unsere eigenen Versuche haben bis zu einem Gehalt von $500 \mu \mathrm{g} / 1$ Nitritstickstoff keinen störenden Einfluss gezeigt (Tab.12).

Tab. 12. Störung des Aufschlusses durch Nitrit.

\begin{tabular}{lcc}
\hline $\mathrm{NH}_{4}-\mathrm{H}[\mu \mathrm{g} / \mathrm{l}]$ & $\mathrm{NO}_{2}-\mathrm{N}[\mu \mathrm{g} / \mathrm{l}]$ & $\mathrm{NH}_{4}-\mathrm{N}$, gefunden $[\mu \mathrm{g} / 1]$ \\
\hline 100 & 0 & 100 \\
100 & 10 & 95 \\
100 & 50 & 90 \\
100 & 100 & 100 \\
100 & 500 & 110 \\
\hline
\end{tabular}

Da in Seen nur in Ausnahmefällen mit Nitritgehalten von mehr als $500 \mu \mathrm{g} / \mathrm{l} \mathrm{zu}$ rechnen ist, kann der Einfluss des Nitrits vernachlässigt werden.

\subsection{Nessler-Reagens, zeitliche Stabilität der Farbreaktion}

Vergleichende Versuche mit dem nach KARLGREN [12] modifizierten PhenolatReagens und dem Nessler-Reagens fielen ganz zugunsten des letzteren aus. Obwohl das Phenolat-Reagens die äusserst empfindliche Indophenolblau-Reaktion ergibt, zeichnen sich verschiedene Nachteile ab. So ist seine Herstellung relativ aufwendig. Ferner ist das Reagens im Gegensatz zum Nessler-Reagens nicht haltbar. Es kann nicht fertig gemischt aufbewahrt werden, und der starke Phenolgeruch bleibt für lange Zeit in den Kleidern des Analytikers und in der Luft des Labors hängen. Wird das Nessler-Reagens sorgfältig hergestellt, so ist es möglich, mit derselben Empfindlichkeit, wie sie das Phenolat-Reagens liefert, auch im Bereich zwischen $0-100 \mu \mathrm{g} / 1 \mathrm{NH}_{4}-\mathrm{N}$ zu messen. Die unterste Bestimmungsgrenze liegt bei $10 \mu \mathrm{g} / \mathrm{l}$. Sind sehr kleine Mengen $\mathrm{zu}$ messen, so ist eine Zugabe von z. B. $100 \mu \mathrm{g} / \mathrm{N} \mathrm{NH}_{4}-\mathrm{N}$ empfehlenswert (aufstocken). Man kommt so in den genauern Bereich der Eichkurve, wobei die $100 \mu \mathrm{g} / 1 \mathrm{am}$ Schluss wieder abzuziehen sind.

Tab. 13 und Abb. 6 geben die zeitliche Ausbildung des Ammonium-Quecksilberjodid-Komplexes wieder $\left(100 \mu \mathrm{g} / 1 \mathrm{NH}_{4}-\mathrm{N}+0,1 \mathrm{ml}\right.$ 10prozentige $\mathrm{H}_{2} \mathrm{SO}_{4}$, Messung bei $440 \mathrm{~nm}$ und $100 \mathrm{~mm}$ Schichtdicke, Vergleich gegen destilliertes Wasser).

Tab.13. Zeitlicher Verlauf der Ausbildung des Ammonium-Quecksilberjodid-Komplexes bei der Ammoniumbestimmung in einer mit Schwefelsäure aufgeschlossenen und anschliessend destillierten Probe.

\begin{tabular}{rlrc}
\hline Zeit [Minuten] & Extinktion $[100 \mathrm{~mm}, 440 \mathrm{~nm}]$ & Zeit [Minuten] & Extinktion $[100 \mathrm{~mm}, 440 \mathrm{~nm}]$ \\
\hline 0 & 0,135 & 60 & 0,154 \\
10 & 0,154 & 75 & 0,157 \\
20 & 0,154 & 165 & 0,158 \\
30 & 0,154 & & \\
\hline
\end{tabular}


Nach 10 Minuten Reaktionszeit hat sich die Farbstärke praktisch voll entwickelt und bleibt über Stunden hinaus beständig.

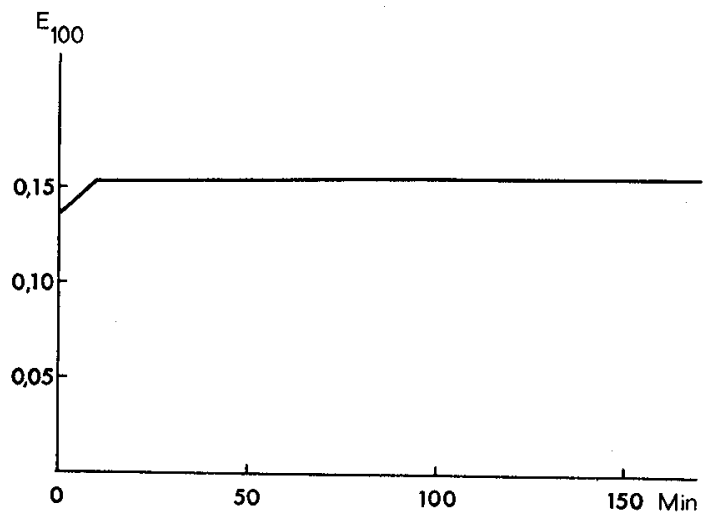

Abb.6. Farbstärke des Ammoniumquecksilberjodid-Komplexes in Funktion der Zeit.

\subsection{Vollständigkeit des Aufschlusses}

Um zu prüfen, ob der Aufschluss unter den angegebenen Bedingungen vollständig verläuft, wurden aus Glycin $\mathrm{C}_{2} \mathrm{H}_{5} \mathrm{NO}_{2}$, Alanin $\mathrm{C}_{3} \mathrm{H}_{7} \mathrm{NO}_{2}$, Argininmonochlorid $\mathrm{C}_{6} \mathrm{H}_{15} \mathrm{ClN}_{4} \mathrm{O}_{2}$ und Tryptophan $\mathrm{C}_{11} \mathrm{H}_{12} \mathrm{~N}_{2} \mathrm{O}_{2}$ (alle mit mindestens 99prozentiger Reinheit) Standardlösungen hergestellt und diese aufgeschlossen. Die gefundenen Werte sind in Tab. 14 zusammengestellt.

Tab.14.

\begin{tabular}{|c|c|c|c|c|c|}
\hline & $\begin{array}{l}\text { Sollwert } \\
{[\mu \mathrm{g} / 1]}\end{array}$ & $\begin{array}{l}\text { Gefundener } \\
\text { Wert } \\
{[\mu \mathrm{g} / 1]}\end{array}$ & & $\begin{array}{l}\text { Sollwert } \\
{[\mu \mathrm{g} / 1]}\end{array}$ & $\begin{array}{l}\text { Gefundener } \\
\text { Wert } \\
{[\mu \mathrm{g} / 1]}\end{array}$ \\
\hline \multirow[t]{4}{*}{ Glycin } & 100 & 100 & \multirow[t]{2}{*}{ Argininmonochlorid } & 100 & 92 \\
\hline & 100 & 120 & & 100 & 92 \\
\hline & 100 & 100 & \multirow[t]{5}{*}{ Tryptophan } & 100 & 80 \\
\hline & 100 & 90 & & 100 & 90 \\
\hline \multirow[t]{3}{*}{ Alanin } & 100 & 98 & & 100 & 90 \\
\hline & 100 & 106 & & 100 & 100 \\
\hline & 100 & 102 & & & \\
\hline
\end{tabular}

Sie zeigen, dass der Aufschluss unter den gewählten Bedingungen (20 Minuten bei etwa $310^{\circ} \mathrm{C}$ und $\mathrm{H}_{2} \mathrm{O}_{2}$ als Oxydationsmittel) für Glycin und Alanin vollständig, für Argininmonochlorid und Tryptophan nahezu vollständig ist.

\subsection{Streuungsversuch}

Zur Ermittlung der Bestimmungsgenauigkeit wurde filtriertes und unfiltriertes Seewasser auf den organischen N-Gehalt untersucht; dabei wurden folgende Resultate erhalten (die verwendeten Begriffe sind nachstehend definiert): 
Tab.15a. Filtriertes Seewasser.

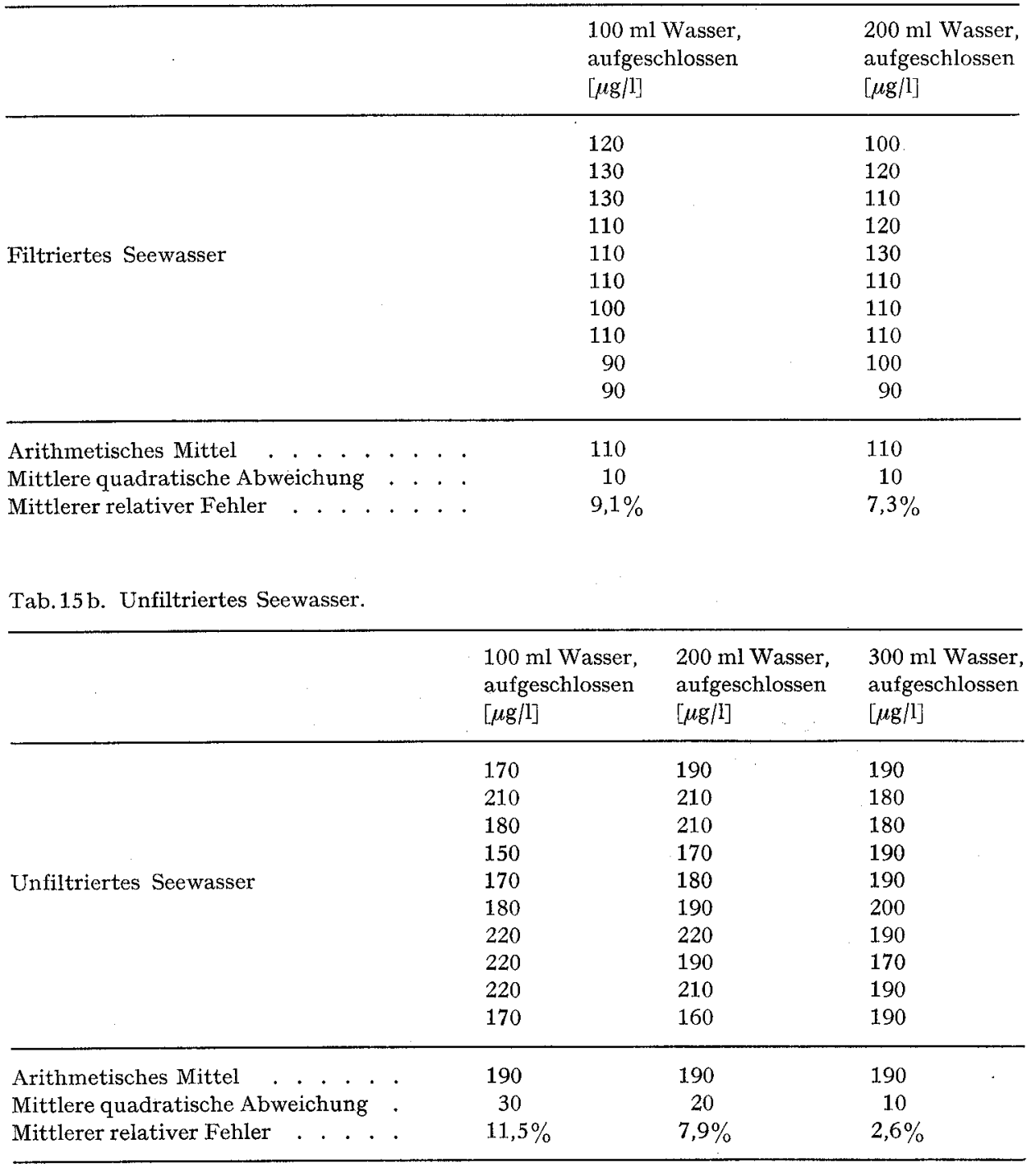

\section{Definitionen:}

Mittlere quadratische Abweichung $=\sqrt{\frac{\sum\left(x_{i}-\bar{x}\right)^{2}}{N-1}}$

Mittlerer relativer Fehler $=\frac{\sum_{1}^{N}|F r|}{N} \%$ 
wobei $\quad x_{i}=$ einzelnes Resultat,

$\bar{x}=$ arithmetisches Mittel,

$N=$ Anzahl Messwerte,

$F r=\frac{\left(x_{i}-\bar{x}\right) 100}{\bar{x}} \%$

Es ist demnach möglich, den organischen Stickstoffgehalt für filtriertes Seewasser mit einer mittleren quadratischen Abweichung von $10 \mu \mathrm{g} / \mathrm{l} \mathrm{zu}$ bestimmen. Bei unfiltriertem Wasser ist der Wert, bedingt durch die Inhomogenität der Probe, Schwankungen unterworfen, die von der verwendeten Wassermenge abhängen.

\subsection{Konservierung}

Strickland und Parsons [30] empfehlen, die Proben bei $-20^{\circ} \mathrm{C}$ einzufrieren, sofern die Analyse für mehrere Stunden aufgeschoben werden muss, ein Verfahren, das von uns nicht näher untersucht wurde. Nach den amerikanischen Einheitsverfahren soll pro Liter Wasser 0,8 $\mathrm{ml}$ konz. Schwefelsäure als Konservierungsmittel zugegeben werden. Unsere eigenen Versuche haben gezeigt, dass es wichtig ist, die Wasserproben möglichst rasch nach der Probenahme aufzuschliessen. Die Zugabe von Schwefelsäure als Konservierungsmittel verändert die Werte der einzelneı organischen Stickstofffraktionen (Tab. 16), so dass die Proben am besten bis zum Aufschluss ohne speziellen Zusatz in Jenaer G20-Glasflaschen kühl aufbewahrt werden. Kann die saure Aufschlusslösung nicht sofort nach dem Aufschluss destilliert werden, so wird sie in möglichst konzentrierter Form (ungefähr $30 \mathrm{ml}$ Lösung) im Kjeldahlkolben verschlossen stehengelassen. Bei dieser Lagerung treten nach unseren Erfahrungen die kleinsten Veränderungen auf.

Tab. 16. Wasser aus dem Bodensee-Untersee aus $10 \mathrm{~m}$ Tiefe, vom Mai 1967, nach verschiedener Standzeit aufgeschlossen.

\begin{tabular}{|c|c|c|c|c|c|c|}
\hline $\begin{array}{l}\text { Standzeit } \\
\text { [Tage] }\end{array}$ & $\begin{array}{l}\mathrm{N} \text {-Gehalt } \\
\text { filtriert }\end{array}$ & $\begin{array}{l}/ 1] \text { neutral } \\
\text { unfiltriert }\end{array}$ & geformt & $\begin{array}{l}\text { N-Gehal } \\
\text { filtriert }\end{array}$ & $\begin{array}{l}\text { 1] } 0,8 \mathrm{ml} \text { kos } \\
\text { unfiltriert }\end{array}$ & $\begin{array}{l}\mathrm{H}_{2} \mathrm{SO}_{4} / 1 \\
\text { geformt }\end{array}$ \\
\hline 1 & 150 & 270 & 120 & 200 & 320 & 120 \\
\hline 2 & 160 & 280 & 120 & 220 & 330 & 110 \\
\hline 3 & $120 ?$ & 260 & 140 & 170 & 290 & 120 \\
\hline 4 & 150 & 260 & 110 & 170 & 300 & 130 \\
\hline
\end{tabular}

Die Zahlen der neutral stehengelassenen Proben sind untereinander kaum als verschieden zu betrachten. Das bedeutet, dass der Aufschluss auch nach 4 Tagen Standzeit noch brauchbare Werte liefert. Schwer erklärbar verhalten sich die angesäuerten Proben. Vergleicht man die beiden bestimmten Zahlen für unfiltriertes und filtriertes Wasser der neutral und sauer gestandenen Proben, so liegen die Werte der letzteren um ungefähr $50 \mu \mathrm{g} / 1$ höher, was jedoch auf die Berechnung des geformten Stickstoffs keinen Einfluss hat. Die Ursache dieser Erhöhung ist unklar.

Ein analoger Versuch mit Vierwaldstätterseewasser zeigte ein ähnliches Resultat. Die Werte der neutral gestandenen Proben blieben bis zum 4. Tag konstant, wogegen die der sauren Proben beständig zunahmen. 
3.11. Anwendung der Methode: N-Gehalte aus dem Vierwaldstättersee, Stelle Kreuztrichter, Oktober 1966

Der von der limnologischen Abteilung der EAWAG jeden Monat auf seine Nährstoffe geprüfte Vierwaldstättersee zeigt an der Stelle Kreuztrichter im Oktober 1966 das in Abb. 7 wiedergegebene Bild. Beim Vergleich des Verlaufs der Nitrat-N- und der Kjeldahl-N-Kurve fällt die qualitative und quantitative Gegenläufigkeit auf. Der Anstieg des geformten Kjeldahlstickstoffs in 7,5 $\mathrm{m}$ und der gleichzeitig bis in diese Tiefe herrschende Nitratschwund zeigen, dass der Nitratstickstoff von den Organismen aufgezehrt wurde. Die biologische Analyse ergab in 7,5 m eine maximale Entwicklung des Phytoplanktons, bedingt durch die quantitative Dominanz von Oscillatoria rubescens. Nach $10 \mathrm{~m}$ Tiefe nimmt der geformte Stickstoffgehalt rasch ab und verläuft parallel zum Nitratstickstoff weiter.

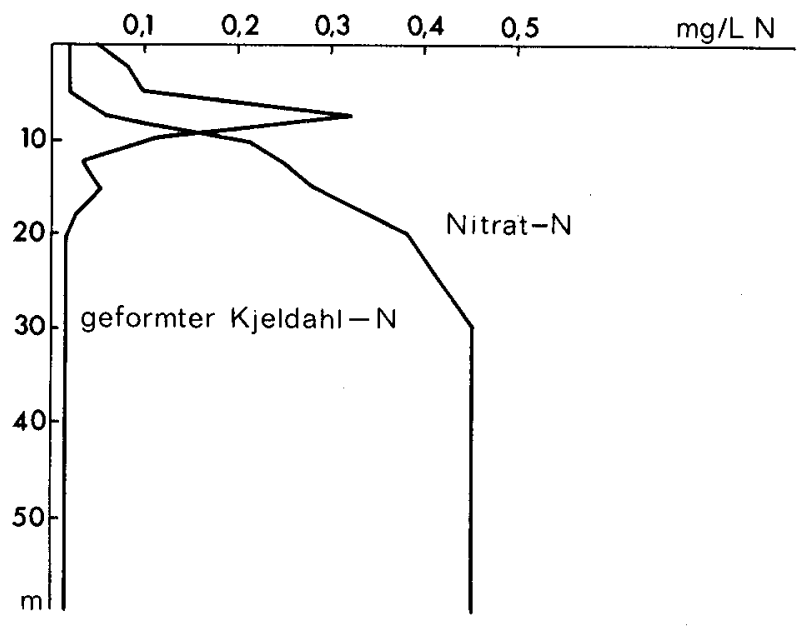

Abb.7. Verlauf von Nitratstickstoff und geformtem Kjeldahlstickstoff im Tiefenprofil des Vierwaldstättersees. Stelle Kreuztrichter, Oktober 1966.

\section{Analysenvorschrift}

Geräte:

a) Kjeldahlkolben aus Glas, Geräteglas G20 von Schott (Jenaer Glas) mit Schliffstopfen, mindestens $250 \mathrm{ml}$ Inhalt.

b) Erlenmeyerkolben mit Schliffstopfen, $200 \mathrm{ml}$ Inhalt.

c) Siedestäbchen (Abb.8), Herstellung durch Ausziehen von dickwandigem Glasrohr, mit Einschmelzung.

d) Verschluss mit zweifachem U-Rohr (Abb.3).

e) Messkolben $100 \mathrm{ml}$.

f) Filterapparat Millipore XX1504700 (Ganzglasausführung) oder ähnliches Gerät.

g) Membranfilter: Die zur Filtration des Seewassers verwendeten Sartorius-Membranfilter MF 50, "gewichtskonstant», Durchmesser $50 \mathrm{~mm}$ (Porengrösse 0,45 $\mu \mathrm{m}$ ), 
werden jeweils mit $200 \mathrm{ml}$ Seewasser vorgespült. Versuche haben gezeigt, dass die Filter nach dieser Behandlung kaum noch organische Stickstoffverbindungen in die Proben abgeben.

h) Destillationsapparat: Soll mindestens $31 \mathrm{~N}$-freies Wasser fassen. Eine Füllung erlaubt die Destillation von rund 12 Proben. Die Schliffstellen dürfen nicht gefettet werden. Da Glasstopfen gern festsitzen, wird die Öffnung für die Zugabe der Natronlauge mit einem Stopfen aus Kunststoff verschlossen.

i) Elektrische Aufschlussbrenner (Salvis-Infrabrenner IBC). Jede Kalotte wird durch ein Blech abgedeckt, das eine kreisrunde Öffnung von ungefähr $60 \mathrm{~mm}$ Durchmesser besitzt. Damit wird eine Überhitzung der temperaturempfindlichen Kjeldahlkolben verhindert.

k) Photometer mit möglichst langen Küvetten (50-100 mm), Filter mit Schwerpunkt bei $440 \mathrm{~nm}$.

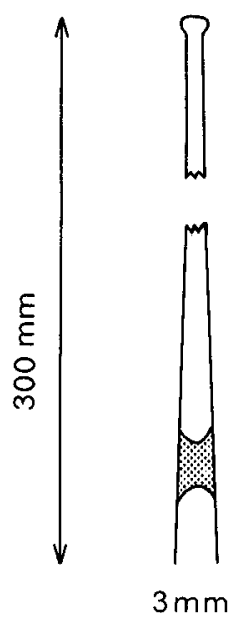

Abb. 8. Siedestab zur Verhinderung von Siedeverzügen beim Abdampfen des Wassers.

Vor dem ersten Gebrauch sind die Geräte a) bis c) mindestens 30 Minuten mit konzentrierter Schwefelsäure auszukochen. Die Kolben werden mit Brunnenwasser und anschliessend mit $\mathrm{N}$-freiem Wasser gespült und ohne weitere Behandlung verschlossen. Vor erneutem Gebrauch werden sie wieder mit N-freiem Wasser ausgespült. Die Kolben werden ausschliesslich für die Stickstoffbestimmung verwendet. Die Verschlüsse mit zweifachem U-Rohr werden vor Beginn einer Untersuchungsserie mit heisser Chromschwefelsäure und mit N-freiem Wasser gespült. Erfolgen mehrere Aufschlüsse nacheinander, so genügt es, die Aufsätze zwischendurch mit $\mathrm{N}$-freiem Wasser zu spülen.

Reagenzien:

$\mathrm{N}$-freies Wasser

Destilliertes Wasser wird unter Zusatz von alkalischer $\mathrm{KMnO}_{4}$-Lösung $(10 \mathrm{~g}$ $\mathrm{KMnO}_{4}+50 \mathrm{~g} \mathrm{NaOH}$ in $1000 \mathrm{ml}$ destilliertem Wasser) unter Luftabschluss (S. 247) 
ein zweites Mal destilliert, wobei der erste N-haltige Anteil verworfen wird. Pro 11 destilliertes Wasser werden $3 \mathrm{ml}$ der obigen Lösung beigegeben.

Nessler--Reagens

$25 \mathrm{~g} \mathrm{HgCl}_{2}$ werden in $375 \mathrm{ml} \mathrm{N}$-freiem Wasser auf dem Wasserbad aufgelöst. Gleichzeitig löst man $50 \mathrm{~g} \mathrm{KJ}$ in $50 \mathrm{ml}$ heissem Wasser. Die abgekühlte $\mathrm{HgCl}_{\mathbf{2}}$-Lösung wird nun unter Schütteln in kleinen Anteilen zur $\mathrm{KJ}$-Lösung gegeben, bis sich der entstehende Niederschlag von rotem Quecksilberjodid gerade nicht mehr löst. Die Menge an ungelöstem Quecksilberjodid darf nur sehr gering sein, da sonst das Reagens unempfindlich wird. Man schüttelt kräftig während 10 Minuten weiter und filtriert dann durch ein Membranfilter. Das klare, gelbe Filtrat wird mit einer Lösung von $108 \mathrm{~g} \mathrm{NaOH}$ in $300 \mathrm{ml}$ Wasser gemischt und auf 11 verdünnt. Zur Steigerung der Empfindlichkeit versetzt man das Reagens mit $5 \mathrm{ml}$ kaltgesättigter $\mathrm{HgCl}_{2}$-Lösüng, lässt über Nacht stehen und filtriert durch ein Membranfilter. Dieser Vorgang wird nochmals wiederholt, wobei aber weitere Zusätze von $\mathrm{HgCl}_{2}$ die Empfindlichkeit des Reagens herabsetzen [26]. Eine Standardlösung mit $0,010 \mathrm{mg} / 1 \mathrm{NH}_{4}-\mathrm{N}$ soll bei Zusatz von $4 \mathrm{ml}$ Nessler-Reagens gegenüber einer gleichbehandelten Probe von $\mathrm{N}$-freiem Wasser eine deutliche Reaktion zeigen.

Schwefelsäure, konz. z.A.

Möglichst N-frei (s. S. 248).

Schwefelsäure $\sim 10$ prozentig

(90 $\mathrm{ml} \mathrm{N}$-freies Wasser $+10 \mathrm{ml}$ konz. $\mathrm{H}_{2} \mathrm{SO}_{4}$ ).

Schwefelsäure $\sim 1$ prozentig

(495 ml N-freies Wasser $+5 \mathrm{ml} \mathrm{konz.} \mathrm{H}_{2} \mathrm{SO}_{4}$ ).

Wasserstoffsuperoxid, 30prozentig

(Perhydrol Merck).

Natronlauge, 32prozentig

$320 \mathrm{~g} \mathrm{NaOH}$ z.A. werden in $680 \mathrm{ml} \mathrm{N}$-freiem Wasser gelöst.

Natriumkarbonatlösung, kalt gesättigt

$20 \mathrm{~g} \mathrm{Na}_{2} \mathrm{CO}_{3}$ (wasserfrei) in $100 \mathrm{ml} \mathrm{N}$-freiem Wasser lösen.

Ammonium-Stammlösung

$4,717 \mathrm{~g}\left(\mathrm{NH}_{4}\right)_{2} \mathrm{SO}_{4}$, bei $100^{\circ} \mathrm{C}$ getrocknet, werden zusammen mit $2 \mathrm{ml} \mathrm{konz.} \mathrm{H}_{2} \mathrm{SO}_{4}$ $\mathrm{N}$-frei auf $1000 \mathrm{ml}$ gelöst.

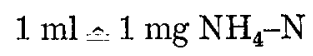

\section{Arbeitsvorschrift:}

Die Proben sollen möglichst bald nach der Probenahme aufgeschlossen werden.

\section{Aufschluss}

100,200 oder $300 \mathrm{ml}$ gut aufgeschütteltes Seewasser werden in einem Kjeldahlkolben mit 2,0 ml konz. Schwefelsäure versetzt und auf dem Infrabrenner bei Höchsttemperatur abgedampft. Beim ersten Auftreten von $\mathrm{SO}_{3}$-Dämpfen werden die Kolben 
weggestellt und sofort mit den gereinigten Aufsätzen verschlossen, wobei der äussere Bogen mit $5 \mathrm{ml}$ 1prozentiger $\mathrm{H}_{2} \mathrm{SO}_{4}$ gefüllt ist. Dadurch wird die Luft, die beim $\mathrm{Ab}-$ kühlen in den Kolben gesogen wird, gewaschen. Nach dem Erkalten werden 3 Tropfen Perhydrol zur Aufschlusslösung gegeben. Um während des Aufschlusses N-Verluste zu vermeiden, wird nun der innere Bogen ebenfalls mit $5 \mathrm{ml}$ 1prozentiger $\mathrm{H}_{2} \mathrm{SO}_{4}$ gefüllt. Schliesslich wird auf dem jetzt tiefer eingestellten Infrabrenner während 20 Minuten bei $310^{\circ} \mathrm{C}$ aufgeschlossen. Die Infrabrenner sind vorher mit einem Quecksilberthermometer, das im Kjeldahlkolben in $2 \mathrm{ml}$ Schwefelsäure steht, zu eichen. Nach dem Erkalten wird die Flüssigkeit des inneren Bogens zum Aufschlussgut gegeben, das Rohr mit $5 \mathrm{ml} \mathrm{N}$-freiem Wasser nachgespült und der Siedestab quantitativ mit 2 mal $5 \mathrm{ml} \mathrm{N}$-freiem Wasser abgespült, so dass am Schluss nicht mehr als $20 \mathrm{ml}$ Flüssigkeit im Kjeldahlkolben zurückbleiben. Um durch die Berührung des Siedestabs mit der Hand keine N-Verbindungen in die Probe zu schleppen, sind entweder die Hände unmittelbar vorher zu waschen, oder es wird noch besser mit hauchdünnen Plastikhandschuhen gearbeitet (s. S. 251), welche nachher weggeworfen werden. Der Kolben wird mit dem Stopfen verschlossen. Da auch die Blindproben $(2 \mathrm{ml}$ konz. $\mathrm{H}_{2} \mathrm{SO}_{4}+3$ Tropfen $\mathrm{H}_{2} \mathrm{O}_{2}$ ) einer gewissen Streuung unterliegen, ist es wichtig, einen guten Mittelwert zu erhalten. Auf 10 Seewasseraufschlüsse werden daher mindestens 2 Blindproben mitbehandelt. Sind somit beispielsweise 42 Proben aufzuschliessen, so werden 9 Blindwerte angesetzt, aus welchen dann das Mittel errechnet wird.

Ansetzen der Typreihe

1,00 $\mathrm{ml}$ Typlösung $\left(1 \mathrm{ml} \bumpeq 1 \mathrm{mg} \mathrm{NH}_{4}-\mathrm{N}\right)$ wird im Messkolben mit $\mathrm{N}$-freiem Wasser auf $100 \mathrm{ml}$ aufgefüllt. Von dieser Lösung wird nach folgender Tabelle in Erlenmeyer-Schliffkolben abpipettiert:

$$
\begin{aligned}
& 0 \mathrm{ml} \bumpeq \quad 0 \mu \mathrm{g} / 1 \mathrm{NH}_{4}-\mathrm{N} \\
& 0,3 \mathrm{ml} \bumpeq 30 \mu \mathrm{g} / 1 \mathrm{NH}_{4}-\mathrm{N} \\
& 0,5 \mathrm{ml} \bumpeq 50 \mu \mathrm{g} / \mathrm{l} \mathrm{NH}_{4}-\mathrm{N} \\
& 1,0 \mathrm{ml} \bumpeq 100 \mu \mathrm{g} / 1 \mathrm{NH}_{4}-\mathrm{N} \\
& 3,0 \mathrm{ml} \bumpeq 300 \mu \mathrm{g} / 1 \mathrm{NH}_{4}-\mathrm{N} \\
& 5,0 \mathrm{ml}=500 \mu \mathrm{g} / 1 \mathrm{NH}_{4}-\mathrm{N}
\end{aligned}
$$

Sehr genau abmessen. Sodann auf $100 \mathrm{ml}$ mit N-freiem Wasser ergänzen (Differenz zu 100, im Messzylinder abmessen). Da die Proben in eine saure Vorlage destillierf werden, erfolgt auch bei der Typreihe die Zugabe von $0,1 \mathrm{ml} 10$ prozentiger $\mathrm{H}_{2} \mathrm{SO}_{4}$. Eine Destillation der Typreihe erübrigt sich, da der von der Schwefelsäure herrührende Fehler sehr klein ist.

\section{Destillation:}

Die Destillationsapparatur wird mit $\mathrm{N}$-freiem Wasser gefüllt und pro Liter mit $2 \mathrm{ml}$ Sodalösung versetzt. Es wird nun so lange vordestilliert, bis Dampf und Apparatur N-frei sind. Zusätzlich erfolgt ein Auswechseln des Kjeldahlkolbens und eine alkalische Destillation (Zugabe von $10 \mathrm{ml} 32$ prozentiger $\mathrm{NaOH}$ ) von $70 \mathrm{ml}$ (s. S. 251). Anschliessend werden der Kjeldahlkolben mit der aufgeschlossenen Probe und die saure Vorlage ( $30 \mathrm{ml} \mathrm{N}$-freies Wasser $+0,1 \mathrm{ml} 10$ prozentiger $\mathrm{H}_{2} \mathrm{SO}_{4}$ ) an die Apparatur angeschlossen, $10 \mathrm{ml} 32$ prozentige $\mathrm{NaOH}$ zugefügt und bis zur 100-ml-Marke der 
Vorlage destilliert. Das Destillat wird in den mit N-freiem Wasser gespülten Erlenmeyerkolben gegeben, der sofort wieder verschlossen wird. Sobald alle Proben destilliert sind, erfolgt die Zugabe von $4 \mathrm{ml}$ Nessler-Reagens. Nach 20 Minuten wird die entwickelte Farbe im Photometer bei $440 \mathrm{~nm}$ gegen destilliertes Wasser gemessen. Für jede Probenserie wird gleichzeitig eine Typreihe hergestellt und anhand der daraus erhaltenen Eichkurve der N-Gehalt der Proben ermittelt.

Der aus den Blindproben errechnete mittlere Blindwert wird sodann von den Resultaten der Proben subtrahiert. Liegt der N-Gehalt der Probe über $500 \mu \mathrm{g} / \mathrm{l}$, so wird das Destillat verdünnt.

Angabe des Resultates auf $0,01 \mathrm{mg} / \mathrm{l}$ genau.

\section{Zusammenfassung}

Für die serienmässige Mikrobestimmung von organischem Stickstoff in Seewasser wurde der Kjeldahlaufschluss und die anschliessende Destillation kritisch geprïft. Der Wirkung von Katalysatoren wurde diejenige von Wasserstoffsuperoxid gegenübergestellt, welches in unserem Fall als Oxydationsmittel vorzuziehen ist. Nitrit stört die Bestimmung kaum, wogegen grössere Mengen an Nitrat mit zunehmender Aufschlussdauer zu erheblichen Verlusten führen. Die beim Aufschluss evtl. auftretenden Stickstoffverluste oder eine durch die verunreinigte Aussenluft bedingte $\mathrm{N}$-Zunahme werden durch Sperrvorrichtungen ausgeschaltet (Abb.3). Weiter wird auf meist wenig beachtete Störeinflüsse wie Blindwert der verwendeten Aufschlussschwefelsäure oder von Stickstoffverbindungen an den Händen hingewiesen. Nach der Destillation in eine angesäuerte Vorlage wird der N-Gehalt der aufgeschlossenen Probe mit Nessler-Reagens kolorimetrisch bestimmt. Die mittlere, quadratische Abweichung beträgt für unfiltriertes Seewasser bei einem Einmass von $100 \mathrm{ml}$ Wasser 30 $\mu \mathrm{g} / \mathrm{l}$, bei $200 \mathrm{ml}$ Wasser $20 \mu \mathrm{g} / 1$ und bei $300 \mathrm{ml}$ Wasser $10 \mu \mathrm{g} / \mathrm{l}$, für filtriertes Wasser (100 oder $200 \mathrm{ml}) 10 \mu \mathrm{g} / \mathrm{l}$.

\section{RÉSUME}

En vue de la micro-détermination de l'azote organique contenu dans les eaux de lacs, la méthode de destruction selon $\mathrm{Kj}$ jeldahl et la distillation ultérieure ont été analysées critiquement. Une comparaison a été faite entre des catalyseurs et l'effet obtenu avec l'eau oxygénée, prise comme moyen d'oxydation donne un résultat préférable dans ce cas. Les nitrites n'influencent guère la détermination, alors que des quantités plus importantes de nitrates entraînent des pertes considérables lórs de destructions prolongées. Les pertes d'azote qui pourraient se manifester en cours de destruction, ou l'augmentation en azote causée par l'air ambiant pollué sont exclues grâce à des dispositifs d'obturation. En outre, on relève certains effets perturbateurs généralement négligés, tels que la "valeur à blanc» de l'acide sulfurique utilisé pour la destruction ou celle de composés azotés qui imprégne les mains. Après distillation dans une solution acide, la teneur en azote de l'échantillon analysé est déterminée colorimétriquement avec le réactif de Nessler. L'écart type moyen pour l'eau de lac non filtrée est de $30 \mu \mathrm{g} / \mathrm{l}$ pour $100 \mathrm{ml}$ d'eau, de $20 \mu \mathrm{g} / 1$ pour $200 \mathrm{ml}$ est de $10 \mu \mathrm{g} / 1$ pour $300 \mathrm{ml}$ d'eau, pour l'eau filtrée $(100$ ou $200 \mathrm{ml})$ il est de $10 \mu \mathrm{g} / \mathrm{l}$.

\section{SUMMARY}

The Kjeldahl digestion and the subsequent distillation was subjected to a critical analysis for the microdetermination of organic nitrogen contained in lake water. A comparison was made between catalysts and the effect obtained, with hydrogen peroxide used in preference, in this case, 
as an oxidant. Nitrite hardly affects determination whereas larger amounts of nitrate entail considerable losses during longer digestion time. Any loss of nitrogen likely to occur in the course of an analysis, or increased nitrogen due to polluted air, are excluded with U-Tubes and packing fluid (Fig. 3). Moreover, a number of disturbing effects usually unnoticed are revealed, namely the blank value of sulfuric acid used for determination and or that of ammonium on hands. After distillation in an acidified receiving-flask (Fig.4), the nitrogen content of the sample is determined colorimetrically with the Nessler reagent. The average square deviation for unfiltered lake water is $30 \mu \mathrm{g} / 1$ for $100 \mathrm{ml}$ of water, $20 \mu \mathrm{g} / 1$ for $200 \mathrm{ml}$ and $10 \mu \mathrm{g} / 1$ for $300 \mathrm{ml}$ of water for filtered water $(100$ or $200 \mathrm{ml})$ it is $10 \mu \mathrm{g} / \mathrm{l}$.

\section{LITERATURVERZEICHNIS}

[1] Apostolacke S., Photometric Determination of Ammonia with Nessler Reagent, Rev. Chim Bukarest. 13, 615 (1962), Analyt. Abstr. 10, 3653 (1963).

[2] BREнM J., Untersuchungen über den Aminosäurehaushalt holsteinischer Gewässer, insbesondeve des Pluss-Sees, Arch. Hydrobiol. Suppl. 32, 313 (1967).

[3] Burck H.C., Kolorimetrische Mikro-Kjeldahl-Methode mit divekter Nesslerisation zur routinemässigen Stickstoffbestimmung, Mikrochim. Acta 200 (1960).

[4] Deyer B., Hamence J.H., The Determination of Nitrogen in Mixed Fertilizers Containing Nitrates and Chlorides, Analyst 63, 866 (1938).

[5] Dudova N., Organischer Stickstoff in Gegenwart von mineralischem Stickstoff, Gidrokhim. Mater. 30, 164 (1960), Chem. Abstr. 56, 281 e (1962).

[6] Fleck A., Munro H.N., The Determination of Organic Nitrogen in Biological Materials. A Review, Clin. chim. Acta 11, 2 (1965).

[7] Galanos D.S., Kapoulas V.M., A Rapid Method for the Determination of Organic Nitrogen and Phosphorus Based on a Single Perchloric Acid Digestion, Analytica chim. Acta 34, 360 (1966).

[8] GonzenBach W., Wissenschaftiche Erforschung des Zürichsees in den Jahren 1929-1933, (Selbstverlag der Wasserversorgung, Zürich 1936).

[9] Hamilton P.B., Amino-Acids on Hands, Nature 205, 284 (1965).

[10] Jönsson E., The Determination of Kjehldahl-Nitrogen in Natural Water, Vattenhygien 22, 10 (1966).

[11] KARCHER F.H., Untersuchungen über den Stickstoffhaushalt in Waldseen, Arch. Hydrobiol. 35 , 177 (1939).

[12] KarLgren L., Modified Hypochlorite-Phenate Method for the Determination of Ammonia in Natural Waters, personal communication.

[13] KJELDAHL J., Neue Methoden zur Bestimmung des Stickstoffs in organischen Körpern, Z. analyt. Chem. 22, 366 (1883).

[14] Kursel H. F., Neue Methoden in der Wasseranalyse, I: Bestimmung des Gesamtstickstoffs, Helv. chim. Acta 78, $180(1935)$.

[15] Mackereth F. J.H., Some Methods of Water Analysis for Limnologists, Freshwater Biol. Ass. Sci. Publ. 21, 40 (1963).

[16] McKenzie H.A., Wallace H.S., The Kjeldahl Determination of Nitrogen. A Critical Study of Digestion Conditions, Temperature, Catalyt and Oxiding Agent, Aust. J. Chem. 7, 55 (1954).

[17] Oro J., Skewes H.B., Free Amino-Acids on Human Fingers. The Question of Contamination in Microanalysis, Nature 207, 1042 (1965).

[1.8] Pavoni M., Die Bestimmung der Biomasse und des Stickstoffgehalts des Planktons und ihre praktische Anwendung (in preparation).

[19] Prochazkova L., Einfluss der Nitrate und Nitrite auf die Bestimmung des organischen Stickstoffs und Ammoniums im Wasser, Arch. Hydrobiol. 56, 179 (1960).

[20] Prochazkova L., Spectrophotometric Determination of Ammonia as Rubazoic Acid with Bispyrazalon Reagent, Analyt. Chem. 36, 865 (1964).

[21] Riley J.P., Skirrow G., Chemical Oceanography 2, 399 (1965).

[22] Rотн H., Bestimmung kleinstev Mengen Stickstoff in organischem Material, Mikrochim. Acta $663(1960)$.

[23] Schach H.G., Die Bestimmung des Ammonium-Ions nach dem Indophenolblau-Verfahren. Limnologica 4, 431 (1966). 
[24] Schмid M., Амвӥнц H., Die Bestimmung geringster Mengen von Gesamtphosphor im Wasser von Binnenseen, Schweiz. Z. Hydrol. 27, 184 (1965).

[25] SchürmanN J., Untersuchungen über organische Stoffe im Wasser des Zürichsees, Vjschr. naturf. Ges. Zürich 109, 409 (1964).

[26] Schweiz. Lebensmittelbuch, 4. Aufl. 199 (Eidg. Drucksachen- und Materialzentrale, Bern 1937).

[27] Starikova N.D., Yarlokovs O.G., Determination of Ammonia and Organic Nitrogen in the Liquid and Solid Phase of Mavine Deposit, Trudy Inst. Okeanol. 67, 157 (1964), Analyt. Abstr. $12,4890(1965)$.

[28] Steyermark A., McGer B.E., Bass E.A., Kamp R. R., Micro-Kjeldahl-Method for Nitrogen in Certain Organic Compounds Containing Nitrogen-Nitrogen and Nitrogen-Oxygen Linkages, Analyt. Chem. 30, 1561 (1958).

[29] Strauch L., Ultramikromethode zur Bestimmung des Stickstoffs in biologischem Material, Z. klin. Chem. 3, 165 (1965).

[30] Strickland J. D., Parsons T. R., A Manual of Sea Water Analysis, Bull. Fish. Res. Board Can. $125,81(1960)$.

[31] Tompkins E. R., KrRk P.L., An Improved Diffusion Method for Total Nitrogen, J. biol. Chem. 142, 477 (1942).

[32] Winkler L.W., Maucha R., Die Bestimmung des Proteidammoniaks in Abwässemn, Arch. Hydrobiol. 30, 122 (1936).

Gegenwärtige Adresse des Verfassers: Marcel Schmid, dipl. Chemiker, School of Public Health, The University of Michigan, Ann Arbor, Michigan 48104, USA.

Separatdruck aus der Schweiz. Zeitschrift für Hydrologie 30, Fasc. 1 (1968) Herausgegeben mit Unterstiitzung der Stiftung der Wirtschaft zur Förderung des Gewässerschutzes in der Schweiz 